\title{
Von den Vegetationsorganen der Selaginella Lyallii Spring.
}

Von H. Bruchmann.

(Mit 28 Abbildungen im Texte, S. Lyallii betreffend.)

Selaginella Lyallii gehört zu den hohen, kräftigen, dichotropen Arten dieser Gattung. Sie besitzt als Hauptachse ein unterirdisch kriechendes und langsam fortschreitendes, mit gleichgestalteten gefransten

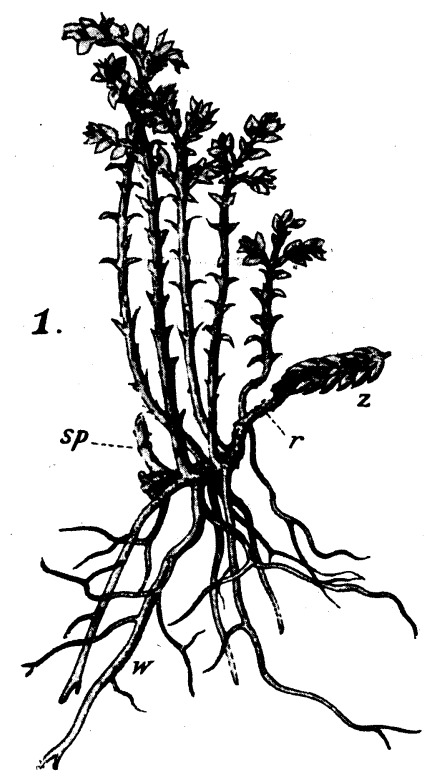

Fig. 1. Junges Pflänzchen durch vegetative Vermehrung aus einem Zweige des Wedels gewonnen.

$z$ Zw eigstück, $r$ Rhizom.

Schwache Vergr. farblosen Blättern besetztes Rhizom. Aus den Seiten desselben treten ab- wechselnd rechts und links aufrechte und gestielte wedelartige Sproßsysteme hervor und an den Auszweigungswinkeln derselben nach abwärts gerichtete Wurzelträger, aus denen starke und reich verzweigte Wurzelsysteme ihren Ursprung nehmen. Während die Wedelstiele gleichgestaltete lanzettförmige Blätter in kreuzenden Paaren geordnet tragen, sind die. Blätter an den fiederartig verzweigten Wedelteilen doppelgestaitig und in dorsiventraler Anordnung aufgereiht (vgl. Fig. 1).

Russow machte zuerst auf ein von den meisten anderen Selaginellen abweichendes Stammscheitelwachstum dieser Art aufmerksam ${ }^{1}$ ), wodurch ich Anregung fand. mich mit der S. Lyallii eingehend $\mathrm{zu}$ beschäftigen. Über die Ergebnisse meiner Untersuchung referierte ich schon vor langer Zeit in einer Generalversammlung des „Thür. Vereins für die gesamten Naturwissenschaften“" ${ }^{2}$ ).

An dem vollständigen Bilde dieser Pflanze fehlte mir aber noch ihre embryonale Entwicklung. Allein meine wiederholten Kulturversuche dieser Pflanze in Gewächshäusern Gothaer Handelsgärtnereien (ein eigenes

1) Russow, Vergl. Untersuchungen der Leitbündel-Kryptogamen. Mémoires de l'Académie de St. Pétersbourg 1873, pag. 176.

2) Ein sehr gekürztes Referat darüber findet sich in Giebeil's Zeitschrift für die gesamten Naturwissenschaften, Berlin, Hempel \& Parey, Dritte Folge, 1877, Bd. I, pag. 524 u. f. 
Gewächshaus stand mir nicht zur Verfügung) zur Erlangung des nötigen Sporenmaterials endeten immer mit dem Absterben der Pflanze.

Inzwischen ist nun von Harvey-(Gibson $\left.{ }^{1}\right)$ in seiner Anatomie der Gattung Selaginella auch S. Lyallii im Bau der. Stengel, der Ligula, der Blätter und der Wurzel eingehend behandelt worden, aber zur Vervollständigung dieses Pflanzenbildes bleibt immer noch manches nachzutragen übrig.

Reiches Material zum eingehenden Studium dieser Pflanze gewinnt man durch ihre leicht $\mathrm{zu}$ erzielende vegetative Vermehrung. Legt man nämlich junge oder alte Zweige der aufrechten wedelartigen Sproßsysteme (mit oder ohne Ähren), welche mit der Mutterpflanze im Zusammenhange bleiben oder auch von ihr getrennt sein können, auf feuchten Sand oder auf gewöhnliche Erde, auch auf Torf oder in Wasser, so kann man nach etwa 6-10 Wochen an jeder Zweigspitze ihre Umbildung $z u$ einem unterirdisch kriechenden Rhizom wahrnehmen (Fig. 2),

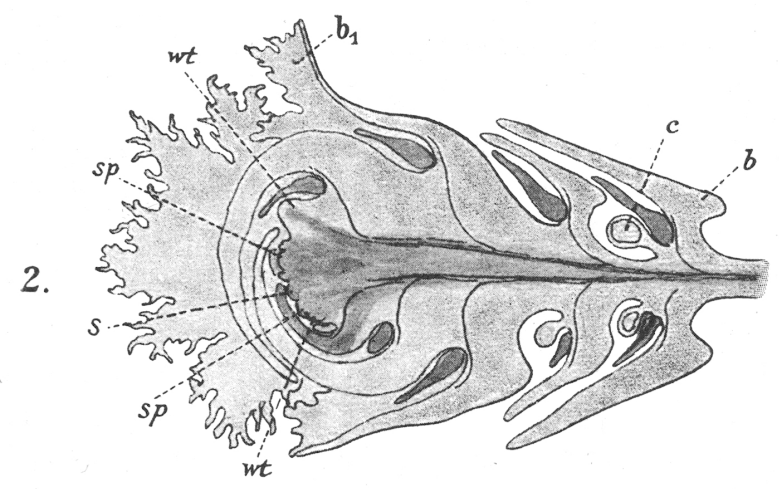

Fig. 2. Ein sich aus einem Blütenzweige bildendes junges Pflänzchen im Längsschnitt. Die fortwachsende Sproßachse stellt die Ausbildung von Sporangien $(c)$ ein, entwickelt an Stelle der ganzrandigen Blälter $(b)$ breite ausgefranste $\left(b_{1}\right)$ und erzeugt die ersten Seitensprosse (sp) und Wurzelträger $(20 t)$. Vergr. 16.

was auch bei anderen dichotropen Arten (z. B. S. umbrosa, S. amoena, S. setosa, S. caulescens, S. erythropus, S. lepidophylla u. a. m.) zu beobachten ist. Ich habe hierüber schon berichtet ${ }^{2}$ ), auch findet sich in der Abteilung der Pteridophyten von Engler's natürlichen Pflanzenfamilien auf pag. 780 (Fig. 408) ein Habitusbild von S. Lyallii mit der Umbildung einiger $Z$ weige in neue junge Pflänzchen vor.

1) Harvey-Gibson, Contributions towards a knowledge of the Anatomie of the Genus Selaginella Spr. Part. I. The Stem (Annals of Botany, Vol. VIII, Nr. 30, June 1894). Part II. The Ligule (Annals of Botany, Vol. X, Nr. 37, March 1897). Part III. The Leaf (Annals of Botany, Vol. XI, Nr. 41, March 1897). Part IV. The Root (Annals of Botany, Vol. XVI, Nr. 63, Sept. 1902).

2) A. a. O., pag. 524. Man vergleiche auch Goebel, Bot.Ztg. 1880, pag. 821, sowie Engler's Natürl. Pflanzenfamilien, Pteridophyten, pag. 666. 
Noch besser als die durch eine vegetative Vermehrung gewonnenen neuen jungen Pflanzen wären für unsere Forschung solche aus einer generativen Vermehrung gewesen. Die embryonale Entwicklung einer dichotropen Form ist noch unbekannt, und es drängt sich hier außer anderen Fragen namentlich die Frage auf, ob die aus der ersten Verzweigung des Embryos entstehenden beiden Gabeläste zu Rhizomen solcher Pflanzen auswachsen werden, was zwar schon im voraus als wahrscheinlich gelten kann. Diese Gabeläste dürften in der gleichen Ebene, in der sie entstehen, sich voneinander wenden und sich vielleicht beide zu Rhizomen ausbilden. Denn die Verzweigungen der Rhizome sowie der ihnen entstammenden Wedel, welche immer in derselben Ebene auftreten, müssen diese senkrecht zu der ersten Dichotomieebene des Embryos gerichtet haben ${ }^{1}$. Leider aber konnte ich diese Frage nicht auf Grund direkter Beobachtung beantworten, da mir zur Untersuchung nur das aus der vegetativen Vermehrung gewonnene Material zur Verfügung stand.

\section{Von den Sprossen.}

1. Das Scheitelwachstum.

Von dem Stammscheitel der S. Lyallii gibt Russow ${ }^{2}$ ) an, daß diesem, wie auch dem einiger anderer Arten dieser Gattung, die durch Größe und Form abweichende Scheitelzelle fehle. Die den Vegetationskegel überziehende Schicht verhalte sich aber insofern abweichend von ler entsprechenden bei. den Phanerogamen, als sie nicht scharf abgegrenzt sei und außer antiklinen auch perikline Teilungen eingehe. Die Anordnung der Zellen des Vegetationskegels sei derartig, daß man sich denselben aus fächerförmig oder richtiger pinselartig nach außen divergierenden Zellenlinien zusammengesetzt denken könne, deren jede an ihrem äußeren Ende fortwachse.

Wir wählen uns zur Prüfung des Scheitelwachstums die Spitzen der im ungestörten Längswachstum begriffenen wedelartigen Sproßsysteme aus. Die Scheitelansicht solcher Vegetationspunkte von oben (Fig. 3) bestätigt Russow's Angabe von dem Fehlen einer bei den Selaginellen zu erwartenden zwei- oder dreiseitigen Scheitelzelle. Sämtliche Zellen eines solchen Organs sehen nach Form und Teilungsweise gleichwertig aus. Auch zeigt ein medianer Längsschnitt (Fig. 4) durch die oberflächliche Zellenlage der Scheitelkuppe neben der antiklinen auch die perikline Teilung. Aber die von Russow angegebenen fächeroder pinselartig nach außen divergierenden und mit besonderen End-

1) Vergl. darüber des Verf. Darlegung bei Sel. spinulosa, pag. 18-22.

2) Russow, a. a. O., pag. 176. 
zellen ausgestatteten Zellfäden zeigt solch Scheitelbild nicht. Vielmehr führen die Zellreihen von unten her auf eine oder einige Zellen der Scheitelmitte, von wo aus das ganze Meristem des Sproßscheitels Erneuerung findet. Ideal wäre es ja, eine einzige feststehende prismatische oder obeliskenförmige Initiale als die Mutterzelle des gesamten Baumaterials der Scheitelspitze anzunehmen, durch deren perikline Teilung nach abwärts Segmente für das zentrale Gewebe abgegeben werden, während ihre antikline Teilung Segmente für die oberflächlichen Zellen erzeugt und die periklinen Segmente der letzteren die Rinde aufbauen. Allein eine solche den Gipfel des Vegetationspunktes einnehmende Zelle tritt nicht besonders hervor. Sie gleicht in Größe, Form und Teilungsweise den benachbarten Zèllen (vgl. Fig. 3 u. 4), weshalb die Herrschaft

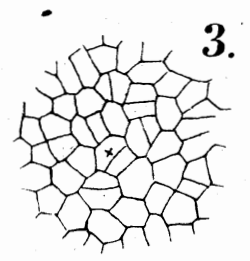

Fig. 3. Scheitel eines Sprosses von oben. Die mit einem Kreuzchen bezeichnete Stelle ist die Scheitelmitte. Vergr. 440.

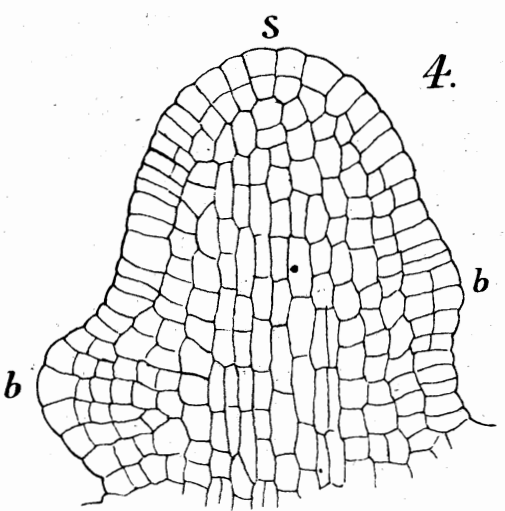

Fig. 4. Längsschnitt durch den Sproßscheitel eines Wedelzweiges. $s$ die Scheitelmitte, $b$ Blattanlagen. Vergr. 440.

solcher Zelle unsicher und wandelbar erscheint und diese durch Beeinflussung mannigfacher Art und durch Wachstumsverschiebungen leicht von einem seitlich abgetrennten Segment im Kampfe um die Scheitelmitte zur Seite geschoben werden wird. Es ist hier die gleiche Art des Wachstums, die ich schon bei S. spinulosa nachgewiesen habe, die auch für die Gattung Lycopodium gilt und am besten kurz als ein Scheitelwachstum mit wandelbaren Initiālen gekennzeichnet wird. Eine Differenzierung der Gewebe tritt spät ein und kann am Sproßscheitel selbst noch nicht erkannt werden.

Die Scheitel der fiederartig verzweigten Nebensprosse unserer Pflanze beleben sich, wie wir wissen, in auffallender Weise, wenn sie einem feuchten Substrate ausgesetzt werden. Die dünnstieligen Sprosse erstarken allmählich, dringen nach abwärts wachsend in das Substrat 
ein und erzeugen an Stelle der grünen, lanzettförmigen, ganzrandigen, ungleichen Blätter in dorsiventraler Anordnung schuppige, zerfranste, farblose, in vier Reihen gestellte Blätter. Kurz, ein Nebensproßscheitel von beschränktem Wachstum wird zu einem Hauptsproß mit andauerndem Wachstum, d. i. zu einem echten Rhizom der Pflanze umgebildet, wodurch also die Pflanze verjüngt und vegetativ vermehrt wird.

Wie nun die größere Feuchtigkeit, welche diese Anregung zur Erneuerung und Umbildung hervorruft, in solch einem abgetrennten wurzellosen Zweige aufgenommen wird, habe ich nicht bestimmt. Vielleicht vermag die ganze Oberfläche des $\mathrm{Z}$ weiges das Wasser aufzunehmen oder es sind besonders die Blattligula und auch die ausgefransten farblosen Blätter selbst zur Wasseraufnahme befähigt.

Fig. 2 stellt einen Ährenzweig in seiner Umformung zu einem Rhizom in Längsschnitt dar. Die letzten Sporangien sind verkümmert, das Weiterwachsen auf dem feuchten Substrat hat sogleich mit den neuen Rhizomblattformen begonnen ( $b_{1}$ in Fig. 2$)$, und nachdem nun aus den von den hinteren Wedelteilen in Lösung zugeführten Baustoffen der umgestaltete Sproß mehrere Millimeter Länge und entsprechende Dicke erreicht hat, geht es alsbald an die Auszweigung der abwechselnd rechts und links in gewissen Zeitfolgen auftretenden Sprosse und Wurzelträger, wobei das Rhizom allmählich fortschreitend immer mehr erstarkt und auch seine Seitenorgane, die zunächst noch jugendliche Formen annehmen, immer kräftiger entstehen läßt.

\section{Die Verzweigung.}

Die erste Verzweigung des Keimlings dieser Pflanze ist sicher eine echte Dichotomie, wie z. B. die der S. spinulosa, S. Martensii u. a. m., während dann aber die weiteren Verzweigungen den Charakter der echten Gabelung verlieren.

Bei S. Lyallii sind die von dem unterirdischen Hauptsproß (dem Rhizom) nach den Seiten abgegebenen Zweige schon bei ihrer Anlage stärker und entwickeln sich auch kräftiger als der Muttersproß, der nur langsam im Substrate fortschreitet, während die aus ihm entstehenden Seitensprosse rege aufwärts streben und in ihrer fiederig verzweigten Form $1 / 2 \mathrm{~m}$ Höhe erreichen können.

Mediane Längsschnitte durch junge Auszweigungsanlagen des Rhizoms (Fig. 5-8) lassen die Aufteilung des Mutterscheitels in zwei gleichwertige Vegetationspunkte, also eine echte Gabelung, nicht erkennen. Es ist aber bei solchem Scheitelwachstum mit nicht besonders gekennzeichneten Initialen schwerer als bei dem mit einer Scheitelzelle, 
den Vorgang der Auszweigung näher zu verfolgen. Wie Treub ${ }^{1}$ ) für die Verzweigung der S. Martensii feststellte, beginnt sie mit der Auflösung der Scheitelzelle. Aber auch hier haben bei dem gleichen Vorgange die Initialen der Scheitelmitte durch eine von der bisherigen abweichende Wachstumsweise die Verzweigung einzuleiten. Als den Anfang einer Verzweigung des Rhizoms darf ein Scheitelbild, das Fig. 5 darstellt, mit Sicherheit ausgegeben werden. Mit einer Verbreiterung des Scheitels in der Auszweigungsebene ist der Anfang gemacht, was nur durch eine veränderte Wachstumsweise des ganzen Gipfelmeristems vor sich gehen konnte. Einmal haben die durch perikline Teilungen an das Scheitelinnere abgetrennten Zellsēgmente das

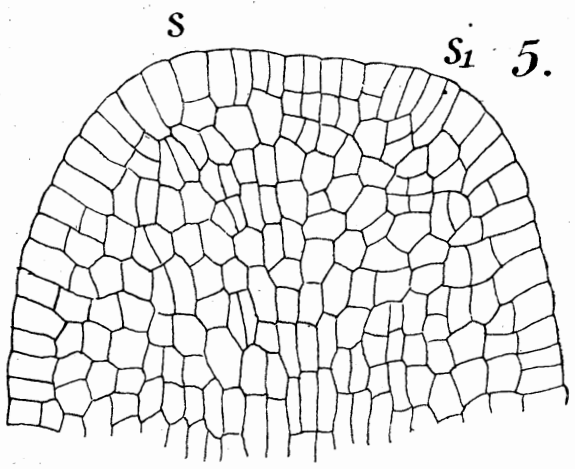

Streben, in der Längsachse zu wachsen, eingestellt und beginnen sich mehr in die Breite zu dehnen. Gleichzeitig teilen sich die Initialen der Scheiteloberfläche fleißig antiklin, namentlich nach den auszweigenden Seiten hin (Figur 5 und 6). Im Sproßinnern aber macht sich alsbald eine solche Anordnung und Teilung der Zellen be-

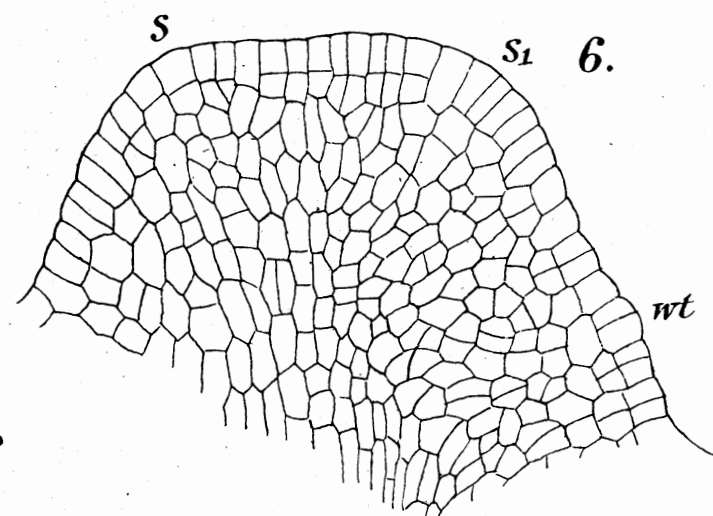

Fig. 5 u. 6. Längsschnitte durch das einen Seitensproß auszweigende Rhizom in den ersten. Entwicklungsstadien. $s$ der Rhizomscheitel, $s_{1}$ der abzweigende Seitensproß, wt Wurzelträgeranlage. Vergr. 440. merkbar, daß hier die divergente Wachstumsrichtung für die neuen Sproßvegetationspunkte zuerst und schon deutlich ausgeprägt entgegentritt, ohe diese äußerlich als̀ gesonderte Höcker erkennbar werden (Fig. 6), worauf denn auch . äußere Zellen an den Stellen einer von innen her erstrebten neuen Wachstumsrichtung lebhaftere perikline und antikline Teilungen eingehen,

1) Treub, Récherches sur les organes de la végétation du Selaginella Martensii, Leiden 1877, pag. 6. 
als benachbarte, und sich als Initialen der neuen Scheitel bekunden (vgl. Fig. 6 u. $7 s$ u. $s_{1}$ ). Die Stelle des erloschenen Scheitelwachstums wird zu einem Sattel zwischen den neuen Vegetationspunkten mit einer für beide gemeinschaftlichen Rinde (Fig. 7 u. 8). Derjenige Zweig, welcher die Hauptachse fortführt, erscheint als der geringere und nimmt

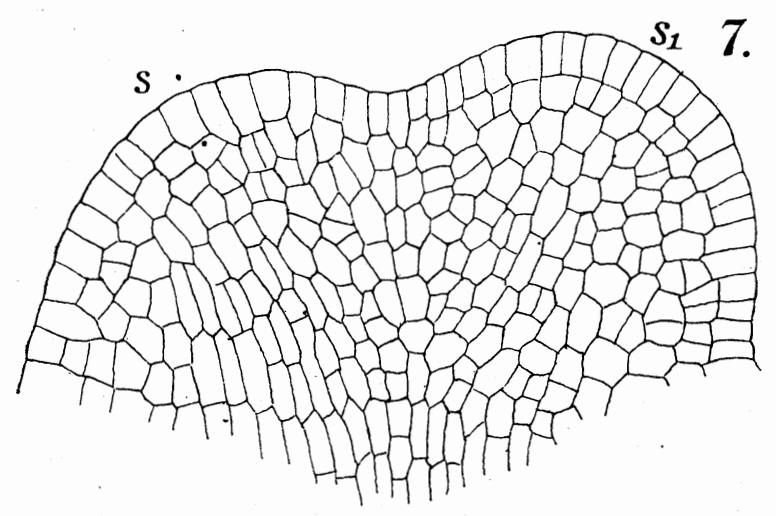
eine von der bisherigen am wenigsten abweichende Richtung an $(s$ in den Fig. 5-8), während die zum aufrechten wedelartigen Sproßsystem bestimmte Auszweigung $\left(s_{1}\right.$ in den Fig. 5-8) die stärkere und im Wachstum bevorzugte ist, die schon früh den schwächeren

Fig. 7 u. 8. Längsschnitte durch das einen Seitensproß auszweigende Rhizom im fortgeschrittenen Entwicklungsstadium. $s$ der Rhizomscheitel, $s_{1}$ der abzweigende und voraneilende Seitensproß, $b$ Blattanlage. Vergr. 440.

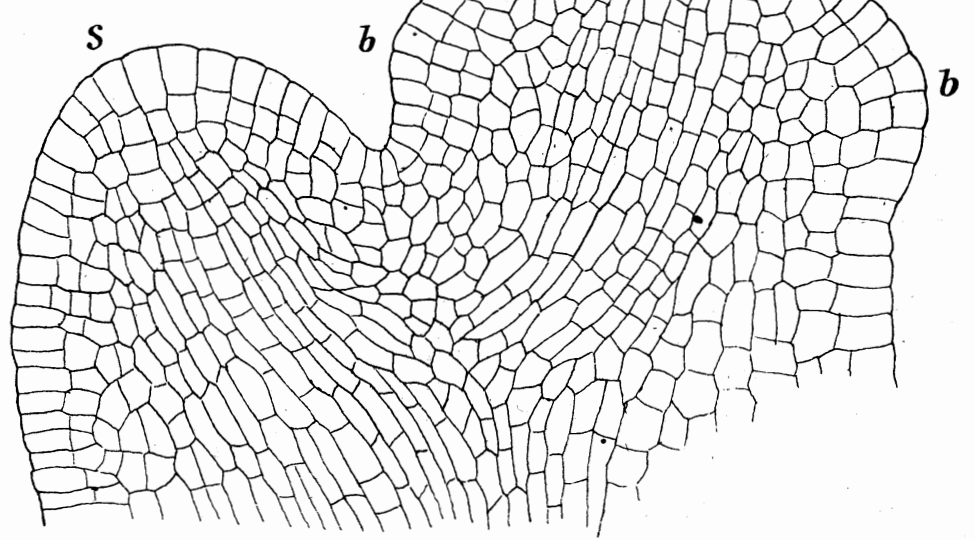

Rhizomast überholt (Fig. 7), auch zuerst beblättert wird (b in Fig. 8), sich dann aus der Verzweigungsebene aufrichtet und im aufrechten und unverzweigten Wachstum mit halbkugelförmiger Scheiteloberfläche den Wedelstiel aufbaut. Der im Rückstande bleibende Rhizomscheitel setzt die kriechende Hauptachse langsam wachsend fort, schreitet aber bald 'zu einer in gleicher Ebene und Form entstehenden weiteren Auszweigung, 
welche an der der vorangegangenen entgegengesetzten Seite den geförderten Seitenast aufstreben läßt. Zuweilen folgen solche Auszweigungen einander so schnell, daß der Rhizomscheitel von den beiden fast aufgebraucht erscheint (Fig. 10).

Wie sind nun diese Verzweigungen des Rhizoms aufzufassen, die doch offenbar als echt dichotome eingeleitet werden? Das Einstellen der bisherigen Wachstumsrichtung und das Auflösen des bestehenden Scheitelwachstums, die Verbreiterung des Scheitels in der neuen zu gewinnenden Verzweigungsebene, die Erstrebung der divergenten Wachstumsrichtung im Meristem von innen her, für welche dann auch im gleichen Sinne zwei Gruppen äußerer Zellen als Initialen der beiden neuen Vegetationspunkte in Arbeit treten, das alles sind Betätigungen echter dichotomischer Verzweigung, welche hier aber nicht als Resultat zwei sich gleichmäßig fortentwickelnde Gabelzweige er-

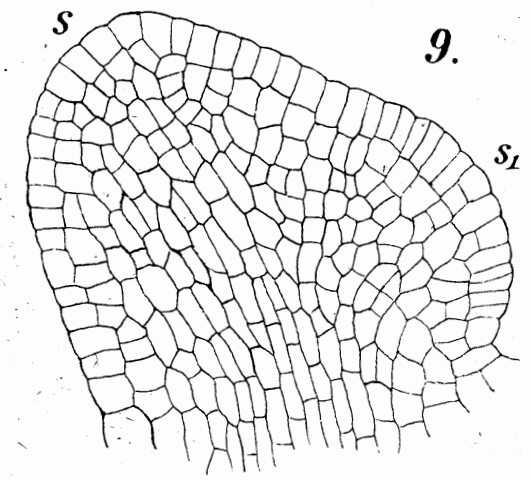

Fig. 9. Verzweigungsbeispiel eines Wedelzweiges. $s$ der voraneilende Hauptzweig, $s_{1}$ ein Seitenzweig. Vergr. 440 .
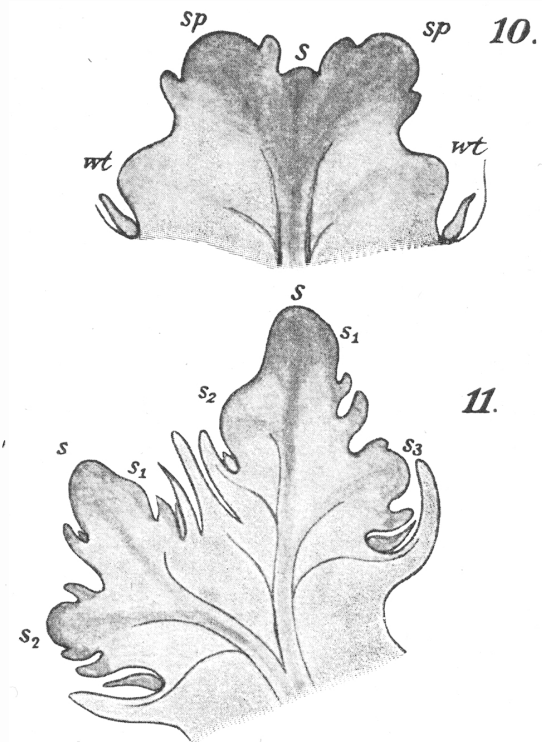

Fig. 10. Ein Beispiel einer Rhizomauszweigung. $s$ der Rhizomscheitel, $s p$ abzweigende Wedelsprosse, zot Wurzelträgeranlagen. Vergr. 52.

Fig. 11. Beispiel einer reichen Anlage von Auszweigungen eines Wedelsprosses. $s$ der im Wachstum voraneilende Sproßzweig, $s_{1}-s_{3}$ seitliche Nebenzweige. Vergr. 52.

zielt, sondern, auf das Nützlichkeitsprinzip abgestimmt, zwei ungleiche Äste, einen schwächeren Ast, der in der Richtung der Hauptachse das Rhizom fortsetzt, und einen kräftig geförderten Seitenast entspringen läßt.

Die Verzweigung der Seitenachsen tritt uns in anderen Bildern als den am Rhizom kennen gelernten entgegen. Wenn diese starken, mit halbkugelförmigem Vegetationspunkt wachsenden Sprosse eine gewisse Höhe erreicht haben, gehen sie auf einmal zur Entwicklung der 
fiederartigen dorsiventralen Spreite lebhafte, in derselben Ebene auftretende Verzweigungen ein. Diese folgen oft so schnell aufeinander, daß ein Längsschnitt durch eine Wedelspitze gleich eine Anzahl junger Auszweigungen aufdeckt (Fig. 11) und man zuweilen auf beiden Seiten des in einer Richtung fortstrebenden Hauptzweiges junge, noch unbeblätterte Sproßtuberanzen antrifft (Fig. 1s, $s_{1}$ u. $s_{2}$ ). Das nähere Studium dieser Auszweigungen zeigt auch hier, daß denselben immer eine Auflösung des Scheitelwachstums und Umgestaltung des Mutterscheitels voraufgeht, daß auch zwei ungleichwertige, in divergenter Richtung fortwachsende Scheitelpunkte erzielt werden (Fig. 9), von welchen aber der die Hauptrichtung fortsetzende (im Gegensatz zur Rhizomverzweigung) der bevorzugte wird und im Wachstum voraneilt ( $s$ in Fig. 9), wogegen der schwächere zurückbleibt und wie ein seitlich entstandener Vegetationspunkt aussieht $\left(s_{1}\right.$ in Fig. 9).

So sind denn diese Verzweigungen der gestielten wedelartigen Sproßsysteme, wie auch die des Rhizoms unserer Pflanze, solche mit dichotomer Anlage, aber von sympodialer Ausbildûng, wie ich sie auch schon an Sel. spinulosa als modifizierte Dichotomie oder als falsche monopodiale Verzweigungen nachwies. Auch die von Sel. Martensii halte ich entgegen der Auffassung von Treub für solche.

Die ersten Wedel an unseren durch eine vegetative Vermehrung entstandenen jungen Pflänzchen sind zunächst noch schwach und erzeugen meist nur eine dreiästige Spreite; bei den weiteren fortwährend kräftiger entspringenden Wedeln werden auch ihre Spreiten größer und reicher verzweigt (Fig. 1).

\section{Vom Bau der Sprosse.}

Nach Dangeards unzulänglicher Beschreibung des Baues der Sprosse dieser Art veröffentlichte Harvey-Gibson seine eingehende Untersuchung darüber. Er fand einen auffallenden anatomischen Unterschied zwischen dem in der Erde kriechenden Rhizom und den aufrechten in der Luft wachsenden Stengeln, welcher so groß sei, daß diese verschiedenen Achsen derselben Pflanze ihrem Baue nach ganz verschiedene Arten darstellen könnten.

Das Rhizom zeigt nach Harvey-Gibson im Querschnitt ein zentrales Bündel, welches das Xylem in der Mitte hat und von den Phloemelementen, der Scheide und einer Lakuna umgeben ist. Dieses zentrale Bündel wird von einem vollständigen hohlen zylindrischen Bündel konzentrisch umschlossen. Letzteres besitzt einen deutlichen Xylemring mit vier äußeren Protoxylemsträngen, welcher von den Phloemund Scheidenelementen von außen und innen konzentrisch eingerahmt 
wird ${ }^{1}$ ). Für die Anlage der Bündel der vom Rhizom aus aufsteigenden Sprosse öffnet sich das zylindrisch hohle Bündel und wird hufeisenförmig. In einigen Fällen umschließt das hohle zylindrische Bündel an Stelle des erwähnten zentralen nur Parenchymzellen ${ }^{2}$ ) (die die letztere Angabe belegende Abbildung ist auch in „Englers natürliche Pflanzenfamilien: Pteridophyten" auf pag. 637 wiedergegeben).

In den starken, über den Boden tretenden unverzweigten Wedelstielen sind eine Anzahl einzelner Bündel (10 und mehr) vorhanden, welche im Querschnitt meist auf einer quadratischen Fläche in drei Reihen verteilt erscheinen und vielfach miteinander anastomosieren. Den vier Eckbündeln werden die Blattspurstränge angefügt. Von den verzweigten dorsiventralen Wedelteilen haben die letzten Zweige zwei außerhalb der Mitte längs den Blattdoppelzeilen verlaufende Bündel. Jedes nimmt die Blattstränge seiner Seite an seinem äußeren Rande auf ${ }^{3}$ ).

Soweit über die bisherigen Ergebnisse der Studien über die Bündel an unserer Art, wobei aber die Auffassung des Rhizombündels durch seine Entwicklungsgeschichte eine wesentliche Erweiterung erfahren muß. Zunächst gilt es hier zu verfolgen, wie der Bündelbau der dorsiventralen oberirdischen Zweige bei einer Überführung durch die vegetative Regeneration in unterirdisch kriechende, radiär gebaute Rhizome mit dem bemerkenswerten abweichenden Bau allmähliche Umgestaltung findet.

Der zur Rhizombildung auf feuchtem Erdreich angeregte dorsiventrale Zweig wird allmählich stärker, seine Blätter stellen sich in vier Zeilen radiär und erhalten breite sehr ausgefränste Spreiten. Die Rinde der Achse erscheint aufgetrieben, und nach einem Wachstum um etwa 3 oder $4 \mathrm{~mm}$ läßt das junge Rhizom schon seine ersten Auszweigungen auftreten. Bis zu dieser Stelle ist auch der Umbau des Zweigbündels in ein Rhizombündel fertig geworden.

Figur 12 stellt im Querschnitt einen dorsiventralen Zweig mit zwei Bündeln dar, von dem eine Umgestaltung zu einem Rhizom den Anfang zu nèhmen hat. Wie nun die Querschnitte durch die jungen Rhizome zeigen, nähern sich die beiden Zweigbündel zunächst der Stengelmitte, dann tangieren sie mit ihren Scheiden und verschmelzen darauf źu einem Bündel mit elliptischem Querschnitt, welches von einer gemeinschaftlichen Bündelscheide und den Phloemelementen umschlossen wird, aber noch zwei seitliche Xylemgruppen besitzt, welche durch

1) Harvey-Gibson, a. a. O., Fig. 76 auf Taf. XI.

2) Ders., a. a. O., Fig. 93 auf Taf. XII.

3) D.e Bary, Vergleichende Anatomie der Vegetationsorgane der Farne usw., Bd. III von Hofmeister's Handbuch der physiol. Botanik, pag. 293. 
Phloemelemente geschieden sind (Fig. 13). Dann treten im weiteren Fortschritt der Rhizomausbildung auch die beiden Xyleme aneinander, zunächst meist U-förmig und endlich ringförmig, und das ganze Bündel, das nunmehr auch kreisrunden Querschnitt erhalten, ist zu einem zylindrischen radiären Rhizombündel umgebaut (Fig. 14). Unter stetiger Zunahme seines Durchmessers und Vermehrung der Bündelelemente
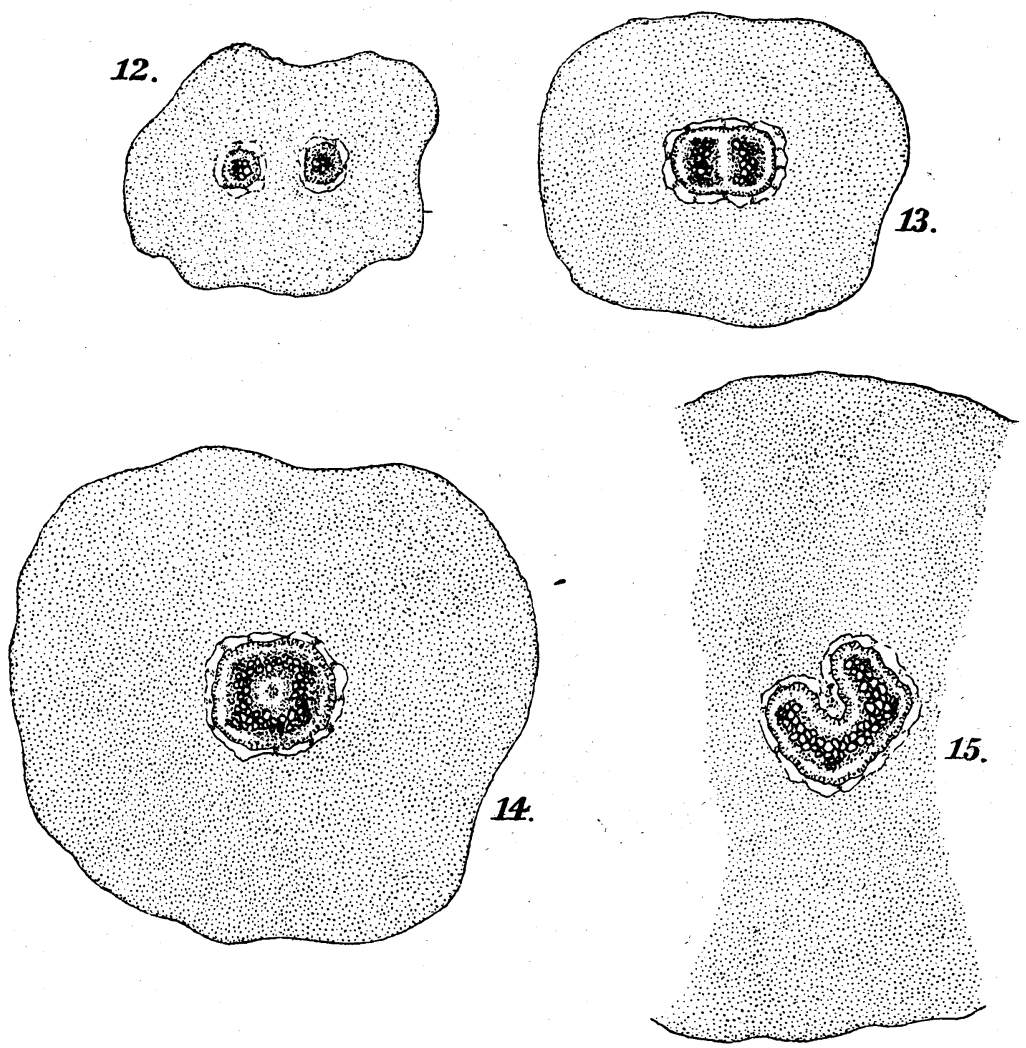

Fig. 12-15. Querschnitte von dem sich unter allmählicher Erstarkung aus einem Wedelzweig entwickelnden Rhizome. Vergr. 52. Die in Fig. 12 dargestellten Bündelelemente treten aneinander und werden dabei reicher an ihren Elementen (Fig. 13), wobei dann das Xylem zu einem Hohlzylinder bei konzentrischer Anordnung der anderen Bündelelemente wird (Fig. 14). Bei Abgabe einer Auszweigung öffnet sich das Bündel und erscheint im Querschnitte hufeisenförmig (Fig. 15). Der

Durchmesser des Rhizoms wächst bei dieser Entwicklung auf das 3-4 fache.

hat das Bündel an Stelle von zwei Protoxylemgruppen es auf vier gebracht (Fig. 17 u. $18 d$ ).

Sehen wir uns die Struktur eines zuerst gewonnenen radiären Rhizombündels einfachster Form näher an (Fig. 16). Seine Mitte wird von einigen Siebröhren eingenommen, in dem Beispiel der Fig. 16 sind 
es die mit $g$ bezeichneten sechs Zellen, die konzentrisch umgeben werden von Phloemparenchym $(f)$, dann dem Xylem $(e)$ mit den peripherischen Gruppen seiner Erstlinge $(d)$, weiter von Phloemparenchym $(c)$, Phloem mit Erstlingen $(b)$ und endlich von einer mehrschichtigen Bündelscheide (Perizykel) (a).

Das Bündel nimmt aber in fortschreitender Entwicklung, wie auch das ganze Rhizom, stetig im Durchmesser zu. Fig. 17 zeigt den Quer-

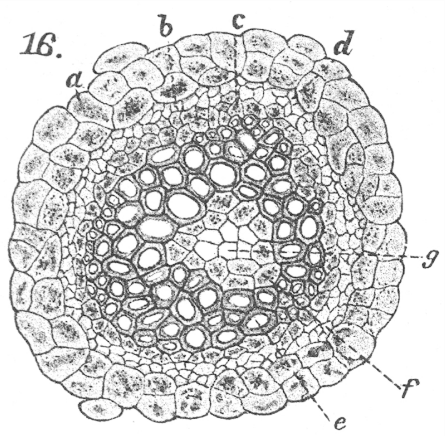
schnitt einer Bündelform, welche zentral einige Parenchymzellen (in der Abbildung drei mit $h$ bezeichnet), die konzentrisch vom Bast und den vorher aúfgezählten Elementen umgeben sind. Diese Parenchymzellen stellen aber die ersten inneren Bündelscheidenzellen vor, welche zur Vervollkommnung des Bündels angebaut werden. Wollte man daran zweifeln, so lehrt es die weitere Ausbildung des Bündels, welche nach einer zentralen Vermehrung der parenchymatischen

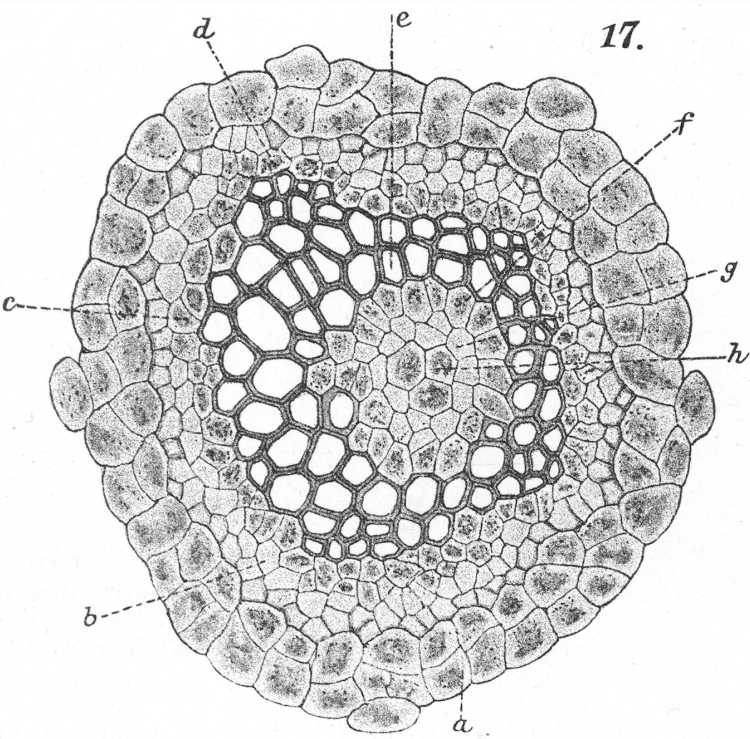

Fig. 16 u. 17. Bündelquerschnitte durch ein erstarkendes Rhizom in der Entwicklung bis zum ersten ringförmigen Bündel. $a$ Bündelscheide, $b$ Phloëm, $c$ Phloëmparenchym, $d$ Protoxylem, $e$ Xylem, $f$ Phloëmparenchym, $g$ Phloëm, $h$ Bündelscheide.

Vergr. 225.
Scheidenzellen endlich auch auf die charakteristische Lakune in der Mitte führt und somit das Bündel zu einem hohlen oder Röhrenbündel ausgebaut hat (Fig. 18). Die Anordnung der Bündelelemente ist die der für Selaginellensprosse charakteristischen Bandoder Plattenbündel, bei welchen sich Phloem und Bündelscheide nebst Lakune symmetrisch zum Xylem anordnen, hier aber mit diesem konzentrisch zum Hohlbündel geschlossen sind. 
Es zeigen somit die Querschnitte der in fortschreitender Entwicklung befindlichen schwachen Rhizome auch eine Fortentwicklung ihres Bündels. Das zylindrische unvollendete Röhrenbündel legt fortgesetzt jedes neu hinzutretende Bündelelement zunächst zentral an und erweitert es dann bei dem Ansetzen des folgenden Elementes trichterförmig und so fort, so daß die einzelnen Elemente des Röhrenbündels in ihrer Entwicklung als ineinander geschobene Trichter angesehen werden können.

Solche Entwicklungsstufe des Rhizoms, die es bis auf die Vollendung des ersten trichterförmigen Röhrenbündels bei einer Zunahme von etwa $4 \mathrm{~mm}$ Länge brachte, hat dabei auch mehr als einen doppelten

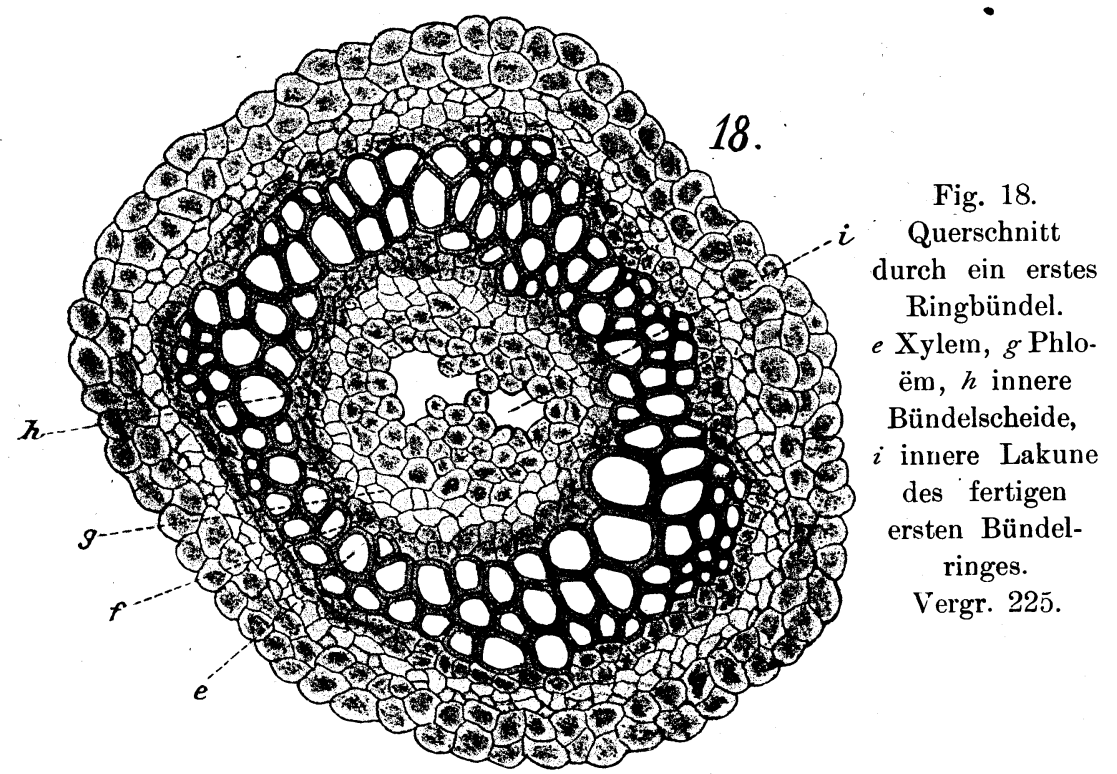

Durchmesser gewonnen (vgl. die Fig. 12, 14 u. 15), und nunmehr zweigt es seine ersten Wedel mit Wurzelträgern ab. Im Querschnitt gesehen zeigt sich das Bündelrohr seitlich vor den Auszweigungen des Rhizoms geöffnet, es erscheint hufeisenförmig mit Zwischenschiebung von Grundgewebe (Fig. 15). Der obere Bündelflügel vereinigt sich zuerst mit dem Bündel des nach abwärts gerichteten Wurzelträgers, worauf dann der untere Bündelflügel mit den Bündeln der aufrechten Achse zusammentritt. Solche Auszweigungen veranlassen in dem Bündelrohre seitliche, abwechselnd rechts und links eintretende Maschenbildungen, welche sich nach jeder seitlichen Auszweigung sogleich schließen.

Mit der weiter fortgesetzten, stetig zunehmenden Erstarkung des Rhizoms geht auch eine weitere Vervollkommnung des Bündelbaues 
Hand in Hand und führt auf eine allmähliche Ausbildung eines zweiten, inneren, konzentrischen Röhrenbündels, welches in seiner vollendeten Form die Fig. 19 und 20 veranschaulichen sollen. Die beiden von Harvey-Gibson dargestellten und in der Einleitung hervorgehobenen Bündelformen des Rhizoms unserer Pflanze sind also keine feststehenden Formen, sondern nur Durchgangsphasen $\mathrm{zu}$ solchen Bündeln, und zwar würde Gibsons Fig. 93 auf Tafel XII die an unsere Fig. 18 anzuknüpfende erweiterte Form darstellen, bei welcher die Bündelmitte ein von einer Lakune umschlossenes Parenchym ausmacht. Dieses zentrale Parenchym leitet als Bündelscheide den Ausbau einer zweiten zentralen hohlen

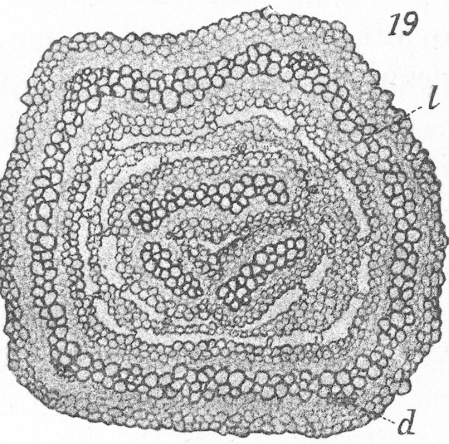
Stele ein. Bei weiterer Vervollständigung folgen darauf Phloem- und Xylemelemente zuerst in zentraler Anlage und dann in trichterförmiger Ausweitung, so daß also in dem fortwachsenden und im Durchmesser zunehmenden Rhizom auch bei der Vervollständigung des zweiten Röhrenbündels am Grunde jedes trichterförmigen Bündelelementes zentral die Anlage des folgenden beginnt. Der durch Harvey-Gibson in Fig. 76 auf Tafel XI dargebotene Bündelquerschnitt stellt eine.Entwicklungsform mit einem halbfertigen inneren Bündel vor, bei der das Xylem

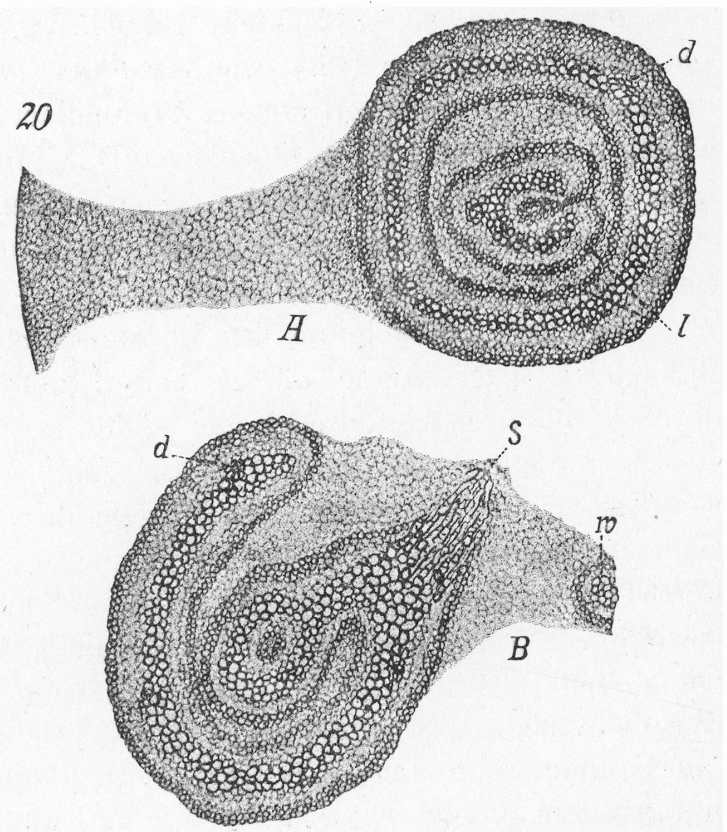

Fig. 19 u. 20. Querschnitte durch die Bündel älterer Rhizome mit zwei fertigen konzentrischen Bündelringen. Fig. 19 Bündelschnitt mit deutlich erkennbaren Lakunen (l). Fig. $20 \mathrm{~A}$ desgl. mit engen undeutlichen Lakunen (l). Fig. $20 B$ dasselbe Bündel bei einer Auszweigung, $s$ Ansatzstelle der Wedelstränge, w Wurzelträgerbündel, welches vorher an dem freien Bündelhorne Anschluß hatte. $d$ Protoxylem. Vergr. 52. 
zunächst zentral, also neu eingestellt war, um darauf in der fortschreitenden Ausbildung den neu einzustellenden weiteren Zuwachs mit den vorherigen, im Querschnitt gesehen, ringförmig zu umschließen.

Besonders starke aus der Heimat dieser Pflanze stammende Rhizome konnte ich leider nicht zur Untersuchung erhalten. Der in Fig. 19 dargestellte Querschnitt entstammt dem Rhizom einer Pflanze aus eigener Kultur, in welchem die Lakunen der beiden konzentrischen Bündelröhren deutlicher zum Ausdruck gekommen sind, als bei dem Schnitte, welchen Fig. 20 zeigt und den ich einem Rhizom entnahm, welches mir gütigst von der Direktion des Königlichen botanischen Gartens zu Kew bei London abgegeben wurde. Die vollkommenste Bündelorganisation, die ich fand, stellen die angeführten beiden Querschnittsbilder in Form von zwei fertigen, konzentrischen Röhrenbündeln dar, die durch ein zwischengelagertes Grundgewebe getrennt sind (Fig. 19 u. 20).

In den Querschnitten des Rhizoms zwischen den aufrechten Auszweigungen zeigt sich der äußere Bündelring geschlossen rundlich, auch viereckig, und er nur führt, wie wir bereits wissen, an seinem äußeren Phloemring wenig hervortretendes Protophloem und an den vier äußeren Ecken des Xylems das Protoxylem. Der innere, zweite konzentrische Ring dagegen variiert gern in seinen Bildern. Sein Xylem erscheint oft durch Bastelemente, auch durch die Scheide und die Lakune getrennt (Fig. 19 u. 20).

Vor der Auszweigung des Rhizoms aber schließt sich der innere Bündelring, auch treten die Xyleme beider Ringe, ferner auch die übrigen Bündelelemente nach der Seite in Verbindung, nach welcher die Auszweigung stattfindet. Diese zeigt nun folgendes Bündelbild, welches wir für die rechte Seite in dem durch Figur $20 \mathrm{~B}$ dargestellten Querschnitt fixierten. Der äußere Bündelring löst sich an dieser Seite durch Dazwischentreten von Grundgewebe auf und das ganze Bündel gewinnt die Form einer Sechs, an die am anfangs noch tiefer gedrückten oberen Bogen die schief abwärts laufende Bündelsäule des Wurzelträgers abgegeben wird. Darauf tritt dieser zurück, und in der Form, wie es die Fig. $20 B$ zeigt, wird an dem unteren Bogen des äußeren Bündels bei seinem Zusammenschluß mit dem inneren Bündelring das Bündel des aufrechten Wedels angeschlossen (Fig. 20Bs, so das Wurzelträgerbündel, welches kurz vorher dem mit $d$ bezeichneten Bündelbogen entsprang). Nach solcher Auszweigung schließt sich das Bündel wieder, um bei der Auszweigung an der linken Seite das Spiegelbild der rechten darzubieten. 
Wenn sich das Rhizom gabelt oder sein Wachstum gestört wird, so daß sein Bau schwächer als vorher einsetzt, greift auch der Bündelbau auf eine niedere Form zurück, um sich dann in der allmählich erstarkenden Achse nach der bekannten und bestimmten Folge auf höhere Formen auszubilden.

Der zusammengesetzte Bündelbau ist, wie gezeigt wurde, im Laufe einer fortschreitenden Entwicklung in gesetzmäßiger Vervollkommnung aus dem einfachen Bau eines durch die Regeneration gewonnenen jungen Rhizoms hervorgegangen. Das Bündel in dem Hypokotyl der Keimpflanze dieser Art, welches wohl mit dem von anderen bekannten Arten übereinstimmen dürfte ${ }^{1}$ ), fasse ich als das ursprüngliche, das Grundoder Urbündel (als Protostele) aller Bündelarten der Selaginellen auf, von welchem durch Umgestaltung alle vorkommenden abgeleitet sind. Tritt aus solchem monarchisch-zentroxylemischen Hypokotylbündel das Protoxylem für die Aufnahme der Blattbündel in geteilten Strängen an die Peripherie des Xylems, so können dann in fortschreitender Ausbildung des Rhizombündelsystems zunächst die Bastelemente und darauf die folgenden Bündelteile zentral angesetzt werden und in trichterförmiger Erweiterung für weitere Anlagen Raum geben und in Form von Röhrenbündeln Vervollständigung finden:

Der Rindenkörper des Rhizoms vergrößert bei der fortschreitenden Ausbildung seinen Durchmesser stetig, was auch ein Vergleich der in gleicher Vergrößerung dargestellten Querschnitte bei den Fig. 12-15 und $20 \mathrm{~A}$ erkennen läßt. Die das Bündel zunächst umschließende Scheide ist bei dünnen Rhizomen einschichtig und bei stärkeren mehrschichtig. Die Zellwände der Scheiden sind nach der Lakune hin kutikularisiert.

Lakunen sind zwar stets vorhanden, doch oft sehr eng und schlecht erkennbar (Fig. $20 l$ ) und führen dann kurze kutikularisierte Lakunarzellen. Sind die Lakunen aber weiter (Fig. $19 l$ ), so haben ihre Zellen die gestreckte, mit den Verdickungsringen versehene Form.

Das ganze Rinden- oder Grundgewebe, auch solches, welches zwischen den konzentrischen Bündeln vorkommt, besteht aus gleichmäßig verdickten Zellen, die interzellularfrei sind und nur in den inneren Rindenteilen vereinzelte kleine Interzellularräume erkennen lassen, nach der Lakune hin sind ihre Außenwände kutikularisiert. Auch die Bündel von Blättern und Wurzelträgern, welche die Rinde

1) Über den Bau der Hypokotyle einiger Selaginellen siehe Bruchmann, Entersuchungen usw., pag. 6 und Anm. 1. 
durchziehen, werden in ihr mit Lakunen umschlossen. Eine eigentliche Hypodermis fehlt dem Rhizom, seine Epidermis ist kleinzelliger als die der Rinde, und ihre Außenwände sind in geringer Dicke kutikularisiert.

Die Blätter des Rhizoms sind gleichgestaltete, chlorophyllfreie, dichtgestellte, mit breiter Basis angeheftete Niederblätter und oft durch die starken abgezweigten Sprosse und Wurzelträger aus ihrer dekussierten Stellung gedrängt. Sie umhüllen schützend den Scheitel des Rhizoms bei seinem Vordringen in den Boden und erhalten dann weiter rückwärts eine ausgebreitete Form mit starkgelapptem, tiefgeschlitztem und ausgefranstem Rande (Fig. 2). Ihr Bau ist sehr einfach und unterscheidet sich wesentlich von dem der oberirdischen Blätter dieser Pflanze. Die äußeren gelappten Randpartien sind im breiten Teile ein-, dann weiter nach der Mitte zu zweischichtig, darauf folgen drei bis fünf und wenig mehr Zellenlagen an der unteren Blattmitte, welches Mesophyll auf beiden Blattseiten einförmige und angeschlossene Zellen hat. Das Bündel, welches nur den unteren Teil der Blattfläche durchzieht, schwachen Formen auch ganz fehlt, ist sehr einfach und besteht oft nur aus einer engen Spiraltracheide, welche von wenigen engen Phloemelementen umgeben ist. Rudimentäre Spaltöffnungen sind auf der Aligularseite der unteren Blattmitte vorhanden. Die Zellen dieser Blätter sind mit dünnem, wässerigem Inhalte versehen, während ihre Ligula dichten schleimigen Inhalt aufweist. Vielleicht haben diese Niederblätter außer dem Schutze der unterirdischen Knospen des Rhizoms auch dazu zu dienen, Feuchtigkeit aufzunehmen und die Pflanzen damit zu versorgen. Auch an den unterirdischen Teilen der aufrechten Sprosse werden zunächst Niederblätter gebildet, darauf Übergangsformen zu den eigentlichen Lichtblättern.

Die aufrechten über den Boden tretenden Sprosse unserer Pflanze sind schon des öfteren eingehend untersucht worden, so von Dangeard, de Bary und namentlich von Harvey-Gibson, weshalb ich nur wenig zu dem bereits Bekannten hinzuzufügen brauche.

Das charakteristische Merkınal des unverzweigten Wedelteiles, das ihn vom Rhizom unterscheidet, ist, wie schon hervorgehoben wurde, die Führung einer Anzahl parallel verlaufender einzelner Bündel. Die bei einer vegetativen Verjüngung aus dem neugebildeten Rhizom zuerst abgezweigten ersten und einfachsten Wedel (man vgl. Fig. 1) zeigen in Querschnitten durch den ganzen Stiel ein zusammen verlaufendes Doppelbündel mit vier Protoxylemgruppen. Mehrfach löst sich dasselbe auch in zwei Einzelbündel mit je zwei Protoxylemgruppen auf. Die 
dorsiventralen Wedelzweige schon der ersten Wedel führen zwei an den Blattdoppelzeilen verlaufende Bündel mit je einer Protoxylemgruppe. Bei den weiteren, allmählich stärker werdenden Wedeln bringen es ihre Stiele bald auf vier gesonderte Bündel, welche, im Querschnitte gesehen, wie die Ecken eines Quadrates gestellt erscheinen. Jedes Bündel führt eine Protoxylemgruppe. In solcher quadratischen Anordnung werden dann bei der weiteren Vervollkommnung der Wedel neue Bündel eingereiht.

Der Wedelstiel ist gefurcht. An die kutikularisierte Epidermis der oberirdischen Sprosse schließt sich auch bei den ersten Wedeln eine gleichfalls kutikularisierte äußere Rinde an. Die innere Rinde ist dünnwandiger und großlumiger. So sind denn die Wedel namentlich durch ihren Bündelbau und durch ihre Blätter von dem Rhizom unterschieden.

\section{Die Wurzelträger und die Wurzeln.}

Harvey-Gibson ${ }^{1}$, der in letzter Zeit der Wurzel der Selaginellen eine besondere Abhandlung gewidmet hat, will von einer Unterscheidung eines Wurzelträgers von der Wurzel nichts wissen, da solche Unterscheidung, seiner Ansicht nach, nicht durch ausreichende morphologische, anatomische oder entwicklungsgeschichtliche Beweise dargetan sei. Der sog. Wurzelträger sei nur ein in der Luft wachsender Teil der Wurzel. Aber schon Nägeli und Leitgeb ${ }^{2}$ ) bewiesen aus der Entwicklungsgeschichte und dem Bau dieser in Frage stehenden Organe ganz überzeugend, daß der Wurzelträger von der Wurzel unterschieden werden müsse und einen anderen morphologischen Wert als die Wurzel habe. Nur behaupteten sie irrtümlich, der Wurzelträger finde sich nicht bei allen Arten der Selaginellen vor, was ihnen auch ebenso falsch von einer Anzahl Autoren bestätigt wurde. Allein in meiner Monographie über Sel. spinulosa ${ }^{3}$ ) war ich zu der Ansicht gekommen, daß es zu dem allgemein gültigen Charakter der Gattung Selaginella gehört, ,ihre Wurzeln nicht direkt, nicht unmittelbar dem Stamme entspringen zu lassen. Es entstehen vielmehr für deren Bildung am Stammgrunde sowohl wie an den Verzweigungswinkeln exogene Organe, in deren Enden die Wurzeln endogen entstehen. Solche Organe, die Stiele oder Träger der Wurzeln, können den Umständen entprechend klein oder groß, schwach oder

1) Harvey-Gibson, Part IV, The Root (Annals of Botany, Vol. XI, Nr. 41, . March 1897).

2) Nägeli und Leitgeb, Beiträge zur wissenschaftl. Botanik, H. IV, 1867.

3) A. a. O., pag. 39 . 
stark, verzweigt oder unverzweigt vorkommen." Dennoch führt HarveyGibson ${ }^{1}$ ) ein direktes Entstehen von Wurzeln aus den Sprossen für eine Anzahl von Arten an, namentlich solche mit unterirdisch wachsenden Rhizomen. Bei zweien der von ihm angeführten Beispiele hebt aber Mager ${ }^{2}$ ) die Anwesenheit deutlich erkennbarer, mehrere Zentimeter langer Wurzelträger hervor. Auch für S. Lyallii, der HarveyGibson ${ }^{1}$ ) gleichfalls die Wurzelträger abspricht, habe ich die Anwesenheit dieser Organe schon früher angegeben ${ }^{3}$ ). Gerade diese letzte Art läßt unterirdische Wurzelträger gut erkennen, welche gewiß nicht als Formen von „Luftwurzeln“ oder „Stützwurzeln“ zu deuten sind; dies kann uns der Umstand lehren, daß sogar an im Boden wachsenden Sprossen Wurzelträger erzeugt werden, wo es einfacher wäre, die Wurzeln direkt aus Rhizomen zu bilden, da eine Verbindung derselben mit dem Boden nicht vermittelt zu werden braucht. Gemäß dem von ihren Vorfahren ererbten Triebe erzeugen die Selaginellen an den Auszweigungsstellen ihrer Sprosse; ob diese in, auf oder über dem Boden auftreten, in kreuzender Ebene zu den Auszweigungswinkeln Wurzelträger. Die Anlage zweier derártiger Träger an jeder Verzweigungsstelle der Sprosse scheint die ursprüngliche Form darzustellen, von welcher aber Übergänge bis zu dem gänzlichen Ausbleiben derselben vorkommen. Recht instruktive Beispiele hierfür bietet die Gruppe der aus kriechender und Ausläufer treibender Basis aufsteigende Wedel bildenden SelaginellenArten. So z. B. erzeugt bei Sel. umbrosa Lem. ein und dasselbe gestielte wedelartige Sproßsystem an den Auszweigungsstellen seines̀ basalen Teiles außer einer schlummernden Knospe je zwei Wurzelträger, von welchen selten ein Träger rudimentär bleibt; dagegen kann in jedem Verzweigungswinkel der Fiedern seines oft über $1 / 4 \mathrm{~m}$ hohen Wedels je eine deutlich erkennbare, nach abwärts gerichtete rudimentäre Anlage eines Wurzelträgers aufgefunden werden, welche durch das Auflegen solcher Wedelteile auf feuchten Boden zur Weiterbildung Anregung findet.

1. Entstehung und Wachstum der Wurzelträger.

S. Lyallii erzeugt nur an den Auszweigungen seines Rhizoms je einen Träger; die oberirdischen, fiederig verzweigten Sproßsysteme lassen an den Auszweigungswinkeln keine Rudimente davon erkennen.

1) A. a. O., pag. 454 .

2) Mager, Beiträge zur Anatomie der physiologischen Scheiden der Pteridophyten. Marburg 1907, pag. 21.

.3) A. a. O., pag. 39. 
Mit jeder jungen Auszweigung des Rhizoms zugleich, seltener etwas später, findet in nächster Nähe des Winkelpunktes der Zweiganlage der Wurzelträger seine exogene Anlage. Eine prismatische Zelle der Sproßoberfläche des embryonalen Meristems wird hier zur Initiale. Sie zeichnet sich meist durch Größe und regere Teilung vor den benachbarten Zellen aus und nimmt die Mitte des durch die Tätigkeit des ganzen Meristems aus der Umgebung hervorgewölbten jungen Wurzelträgerscheitels ein (Fig. 6wt u. 21). Und schon bei dem ersten Auftreten dieser Anlage erhält sie durch eine prokambiale Zerlegung der Rinde unterhalb des Höckers und einer Streckung der Zellen in der Wachstumsrichtung einen Bündelanschluß an die Rhizommitte (Fig. 6 u. 21).

Das Scheitelwachstum dieser Anlage ist anfangs dem eines Sproßscheitels ganz ähnlich, tritt aber bald zurück, und die Anlage wird mehr durch eine interkalare Streckung ihrer Zellen hervorgetrieben. In der Ausbildung, welche Fig. 22 darstellt, hat das Scheitelwachstum bereits sein Ende erreicht, der Scheitel erleidet hierauf eine Umbildung, er verbreitert sich, und mit einer Reihe perikliner Teilungen im Scheitelinnern tritt zugleich eine kappenförmige Anordnung und Auftreibung seiner Zellen ein. Seine Meristemzone wird damit von dem Scheitelgipfel nach rückwärts verlegt und eine interkalare Meristemzone eingerichtet (Fig. 23). In dieser wird nun während des weiteren Wachstums nach und nach endogen die Anlage der Wurzel ausgeführt. Doch ehe wir hierauf näher eingehen, wollen wir vorher das Wurzelwachstum kennen lernen.

\section{Das Scheitelwachstum der Wurzel.}

Dem Vegetationspunkte der Wurzel fehlt die Scheitelzelle, was ich schon an anderer Stelle mitteilte $\left.{ }^{1}\right)$. Dieser Wurzelscheitel stellt, wie ein medianer Längsschnitt desselben zeigt (Fig. 26), einen für die Gattung Selaginella seltenen Wachstumstypus dar, wie ich ihn nur noch an Sel. Preissiana kennen lernte ${ }^{2}$ ), und welcher dem der Gattung Lycopodium nahe kommt.

Die einschichtige Epidermis der Wurzel überzieht als ein scharf abgegrenztes Dermatogen mit nur antiklinen Teilungen ihren Scheitel ( $d$ in Fig. 26) und grenzt den eigentlichen Wurzelkörper von seiner Haube ab. Letztere wächst durch ein eigenes Bildungsgewebe, die

1) Bruchmann, a. a. 0., pag. 28, Anm.

2) Herr Prof. Goebel hatte mir das Spiritusmaterial von Selagin. Preissiana gütigst übērlassen. 
H. Bruchmann,
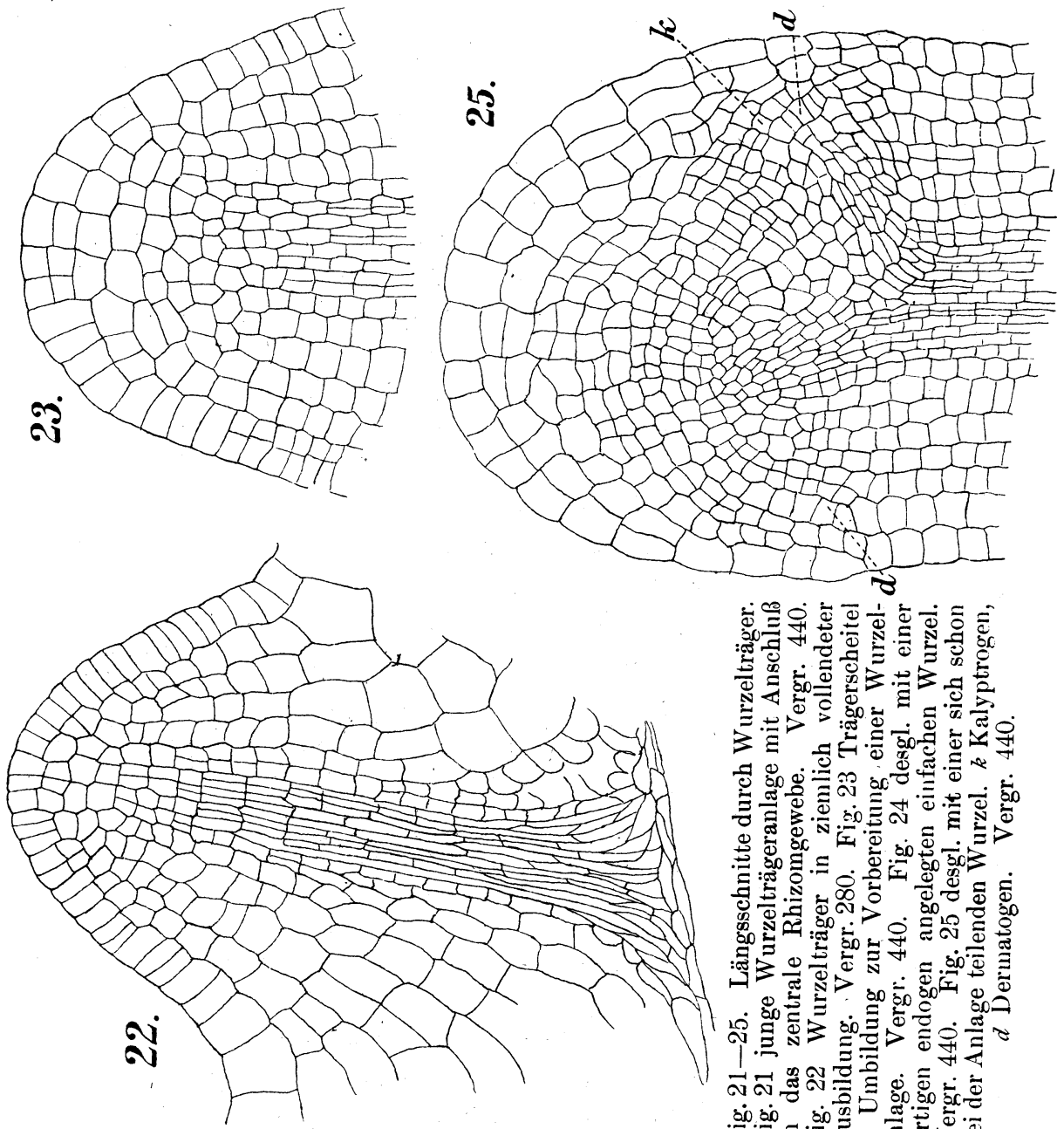

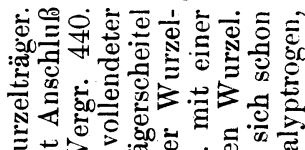

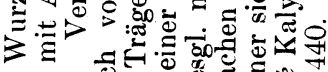
专

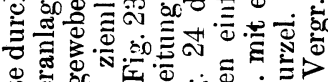

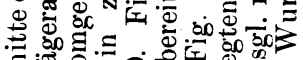

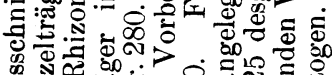
员高:

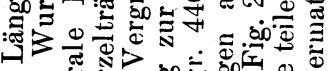
19.

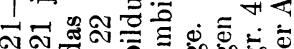

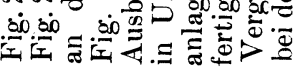
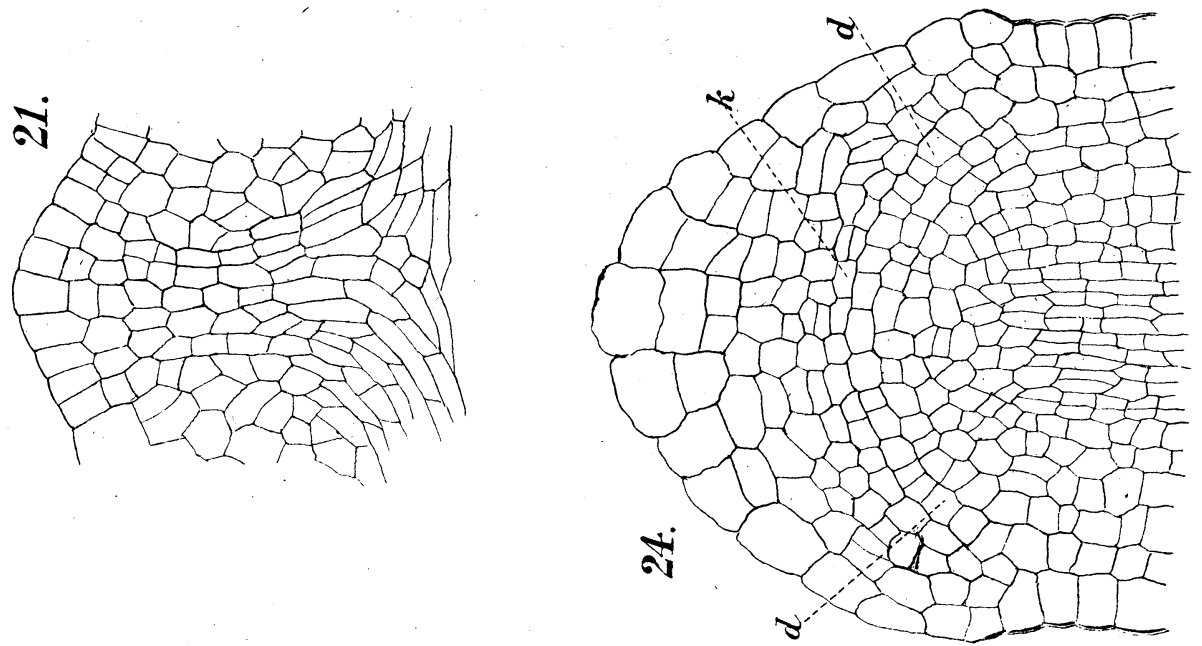
Kalyptrogeninitialen ( $k$ in Fig. 26), welche zum eigentlichen Wurzelkörper in keinerlei genetischer Beziehung stehen. Das Dermatogen ergänzt sich aus eigenen Initialen, wogegen das übrige Meristem der Wurzelspitze, Periblem (pb) und Plerom (pl) scheitelwärts gemeinschaftliche Initialen aufweisen (Fig. 26). Doch kann man am Wurzelscheitel die innere, dem Dermatogen anliegende Schicht manchmal als eine ungeteilte Mantelschicht erkennen, welche scheitelwärts nur antiklin segmentiert und erst seitwärts perikline Teilungen eingeht, wie es z. B. die Figur 28 zeigt. Es führen also die äußeren Periblemschichten scheitelwärts zuweilen auf eine einzige ungeteilte Mantelschicht. Doch zeigen dann wieder andere Fälle, daß aus der in Frage stehenden Schicht auch Zellen durch perikline Teilungen an das Scheitelinnere abgegeben werden (Fig. 26). Somit wachsen Plerom und Periblem aus gemeinsamen Initialen, welche für die äußeren
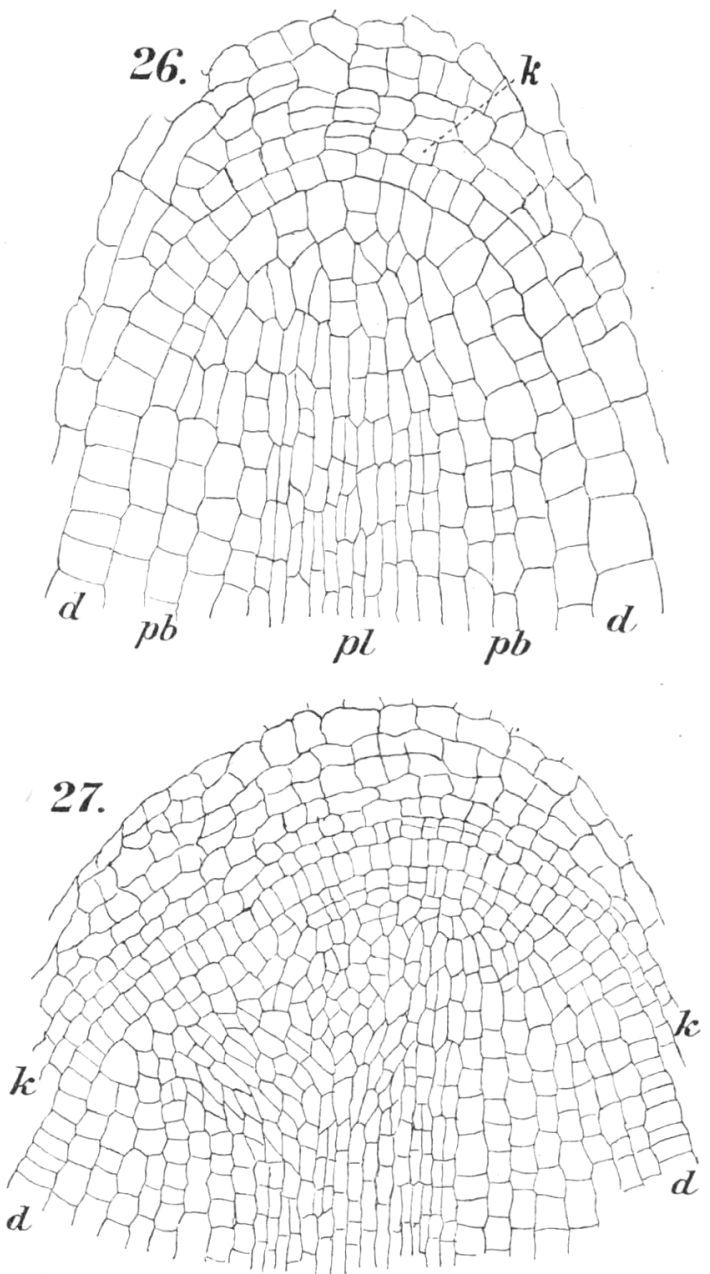

Fig. 26 u. 27. Längsschnitte durch Wurzelspitzen, durch eine einfache (Fig. 26, Vergr. 440) und durch eine sich verzweigende Wurzel (Fig. 27, Vergr. 280).

$k$ Kalyptrogen, $d$ Dermatogen, $p b$ Periblem und pl Plerom.

Periblemreihen zeitweise eine Abgrenzung erkennen lassen. Es gleicht dieser eigenartige Wurzeltypus von Selaginella beinahe dem einer Lycopodium-Wurzel, welche sich nur durch eine weitere auf das Scheitelinnere geführte Abgrenzung des Pleroms vom Periblem unterscheidet. 
Die Wurzeln der S. spinulosa, welche auch ihre Haube unabhängig vom Wurzelkörper aufbauen, besitzen für letzteren nur eine Initialgruppe, somit stellen die Wurzeln von S. Lyallii mit ihren drei Initialgruppen eine Übergangsform von dieser zu denen der Lycopodien mit ihren vier Initialgruppen dar.

\section{Die Verzweigung der Wurzeln.}

Das aus einem Wurzelträger entsprungene Wurzelsystem stellt eine stärkere Hauptwurzel dar, welche vorausstrebend das Erdreich

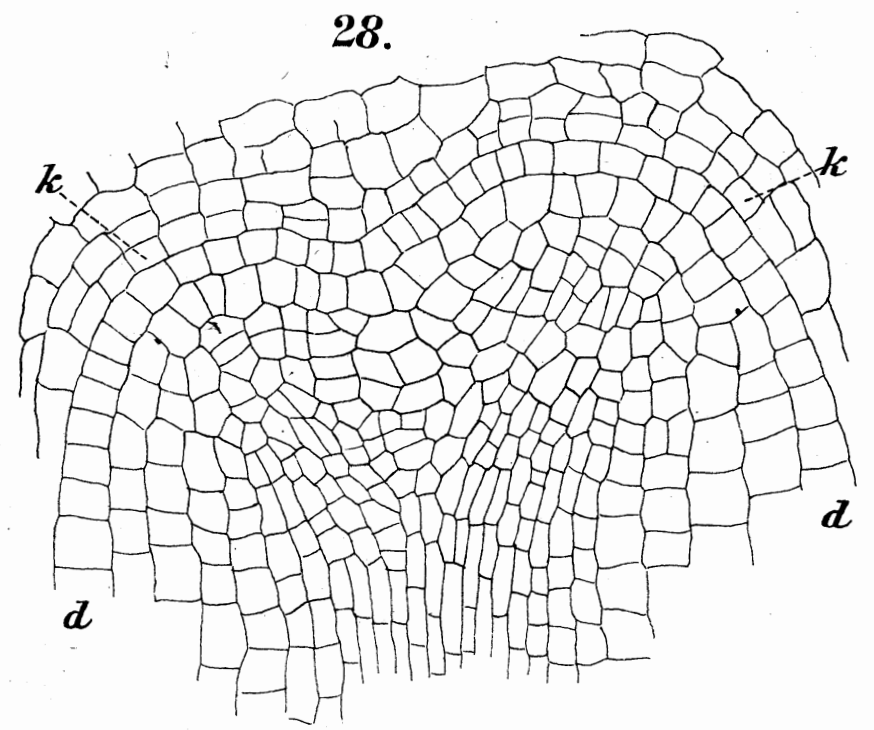

Fig. 28.

Längsschnitt durch eine sich verzweigende Wurzelspitze in fortgeschrittener Entwicklung. $d$ Dermatogen, $k$ Kalyptrogen. Vergr. 440.

durchdringt und seitlich schwächere sich weiter verzweigende Nebenwurzeln abgibt (Fig. 1). Auch das Verzweigungssystem der Wurzel erscheint also in fertiger Entwicklung monopodial $\mathrm{zu}$ sein, doch wird es ebenso, wie das der Sprosse, als pseudomonopodial aufzufassen sein. Die Verzweigung findet nur in der Wurzelspitze statt und tritt, wenn auch mit einiger Unregelmäßigkeit, in sich kreuzenden Ebenen auf. Bei näherem Eingehen auf die beiden Abbildungen medianer Längsschnitte durch junge Verzweigungen zeigt sich eine sehr ungleiche Anlage derselben (Fig. 27 u. 28). In beiden Fällen läßt sich erkennen, $\mathrm{da} \beta$ diese Neugestaltung des Wurzelscheitels zuerst im Innern am deutlichsten ausgeprägt ist. In dem Falle, welchen Fig. 27 darstellt, erscheint es fast, als wenn rechts der Hauptscheitel unverändert fortwachse und an der linken Seite nahe der Spitze eine neue Wurzel seitlich angelegt werde. Allein eine solche monopodial ausgezweigte 
neue Wurzel müßte ja mit neuen Meristemarten endogenen Ursprung finden, was aber nicht der Fall ist. Man kann bei solchen Auszweigungen erkennen, daß von dem achsilen Scheitelmeristem des Wurzelinnern immer ein, wenn auch geringer Teil, zur Seite abgeteilt wird und daß auch die übrigen Meristeme, nämlich das Dermatogen und das Kalyptrogen, von denen der Mutterwurzel abzuleiten sind. Dies findet den besten Ausdruck namentlich im Dermatogen, welches stets in ununterbrochener Folge auf beide neue Scheitel der Verzweigungen übergeführt wird. Man hat es hier mit einer gleichen Verzweigung zu tun, wie sie die Wurzeln der Lycopodien häufig zeigen, mit einer modifizierten Dichotomie in sehr ungleichmäßiger Anlage und Weiterentwicklung der neuen Triebe (Fig. 27 u. 28).

Eine Teilung des Wurzelscheitels in zwei gleichstarke Glieder findet hier auch bei den Nebenwurzeln nicht statt. Aber Sel. spinulosa bietet solch Beispiel dar, bei welchem der Wurzelscheitel auch bei einem Wachstum mit gesonderten Initialen Dichotomie aufweist ${ }^{1}$ ).

\section{Die Anlage der Wurzeln.}

Die Wurzeln entspringen hier, wie bei anderen Arten, endogen in dem interkalaren Meristem der Wurzelträgerspitze. Sie werden gleichsam der Trägerspitze aufgepfropft und erscheinen als eine in derselben Richtung weitergeführte Fortsetzung der Muskelträger. So ist es bei der Gattung Selaginella Regel. Allein, daß ein Wurzelträger unter Umständen auch die Wurzeln seitlich hervortreten läßt, wurde experimentell dargetan ${ }^{2}$ ) und dürfte auch hier möglich sein.

Wenn das Scheitelwachstum des Wurzelträgers eingestellt wird und ein Wachstum durch ein interkalares Meristem aufkommt, gestaltet sich der Vegetationspunkt allmählich zu einer ersten Haube für die darauf scheitelrückwärts auszubildende Wurzelanlage um (Fig. 22 u. 23). Bei den mit einer Scheitelzelle wachsenden Wurzelarten ist ihre Anlage vollzogen, wenn in dem interkalaren Meristeme der Träger eine oder mehrere Scheitelzellen hervortreten. Hier aber ist eine Wurzelanlage nicht so einfach, da für den neuen Wurzelscheitel ein gesondertes Scheitelmeristem aufzubauen nötig wird. Die Wurzel kann als fertig angelegt gelten, wenn die einzellige Dermatogenreihe differenziert ist (Fig. 24 u. 25 $d$ ). In dem embryonalen interkalaren Meristem der. Wurzelträger tritt nach und nach eine aus größeren Zellen bestehende einzellige Schicht hervor, die in der Scheitelkuppe über der Längsachse

1) A. a. O., pag. 28 .

2) Flora, Ergänzungsband 1905, Heft 1, pag. 160 und 205. 
zu einem Kugelgewölbe, im Längsschnitte gesehen aber zu einem Bogen angeordnet ist und sich von dem gemeinsamen Triebe beseelt zeigt, an Stelle der unregelmäßigen Teilungen nur einheitliche antikline einzugehen (Fig. 24d). Diese Differenzierung des Dermatogenmeristems der Wurzelanlage hebt meistens ringsum von den Seiten her an und führt, sich zusammenschließend. scheitelwärts fort aḷmählich auf eine abgerundete Scheitelform. Seitlich wird diese Dermatogenschicht, also die spätere Epidermis der Wurzel, stets auf die Rinde des Wurzelträgers und nicht auf die Epidermis desselben gestellt (Fig. 24d), und mit ihrer Differenzierung ist zugleich die der anderen Wurzelscheitelmeristeme eingetreten, nämlich des Kalyptrogens $(k)$ über der dermatogenen Wölbung und des Meristems für die inneren Histogene unterhalb derselben.

Eine so klare und einfache Wurzelanlage mit nur einer Wurzel, wie sie durch Fig. 24 dargestellt ist, wird nur in sehr dünnen Wurzelträgern gefunden. In stärkeren Trägern tritt zugleich die Anlagè mehrerer Wurzelscheitel auf, wie dies ja auch bei den mit einer Scheitelzelle wachsenden der Fall ist. Aber solche werden hier nie gleich stark hervorgebracht, sondern, wie bei einer Wurzelverzweigung, differenzieren sich neben einer Hauptwurzel zugleich seitlich eine oder auch zwei geringere Nebenwurzeln (Fig. 25). Treten zwei Nebenwurzeln zugleich auf, so werden sie nicht in gleichen Ebenen angelegt. Die Differenzierung mehrerer Wurzelspitzen wird in dem interkalaren Meristem eines starken Trägers schon bemerkbar, bevor das Dermatogen für diese vollständig gesondert worden ist, und zeigt sich zuerst in einer Teilung und einer gesonderten Wachstumsrichtung des Pleroms. So zeigt Fig. 25 die Anlage zweier Wurzelscheitel, bei welchen einige Zellen des Dermatogens entgegen ihrer Bestimmung durch perikline Teilungen dem Kalyptrogen oder dem Periblem Zellsegmente zuführen ( $d$ in Fig. 25).

Mit der Differenzierung einer solchen mit gesonderten Initialen wachsenden Wurzel steht der Wurzelträger von S. Lyallii einzig da und stellt deswegen einen besonderen Trägertypus vor, während er sich weder durch seine Anlage, noch durch die Wachstumsweise oder die geringere Ausbildung von den Trägern einiger anderer Arten unterscheidet.

5. Vom Bau der Wurzelträger und der Wurzeln.

Nicht nur durch die Anlage und das Wachstum sind Wurzel und Wurzelträger unterscheidbar, sondern auch in ihrem Baue ist der Unterschied dieser Organe deutlich ausgeprägt. Schon die äußerliche Prüfung 
eines aus der Verzweigungsstelle des Rhizoms entspringenden Wurzelsystems mit der Lupe läßt den basalen Teil desselben, also den Wurzelträger, welcher bis zur ersten Auszweigung reicht und kaum $1 \mathrm{~cm}$ Länge hat, durch die unterschiedliche Epidermis von der folgenden Wurzel auseinanderhalten. Auch tritt der Unterschied dieser beiden Organe äußerlich durch eine verschiedene Aufnahme von Farbstoffen, z. B. des Safranins, hervor. Bei den meisten anderen Selaginellaarten zeigen ja die Wurzelrhizoide den Anfang der den Trägern aufsitzenden Wurzeln deutlich an, allein die Wurzeln der S. Lyallii sind rhizoidlos. Harvey-Gibson, der ja das Vorhandensein eines Wurzelträgers als besonderes Organ nicht zugibt, hebt hervor, daß S. Lyallii bei anormaler Struktur des Stengels doch normalen Wurzelbau besitze, und bildet in Figur 22 auf Tafel XXI den inneren, die Wurzel nicht charakterisierenden Teil eines Querschnittes ab. Neuerdings hat nun Mager ${ }^{1}$ ) bei seinem eingehenden Studium der Scheiden der Pteridophyten auch der Wurzel der Selaginellen besondere Aufmerksamkeit zugewandt, ihren Bau im primären, sekundären und tertïren Zustande beobachtet und sie von den Trägern abweichend gefunden. Er machte seine Studien an 20 verschiedenen Selaginellaarten. Da unter diesen sich unsere Art nicht befindet, bei der aber als ein besonders hervorzuhebender Fall auch der Träger, wie die Wurzel, ein ganz unterirdisches Dasein hat, so ist hier der Unterschied im Bau dieser Organe besonders hervorzuheben.

Nebeneinandergelegte Querschnitte von Wurzelträger und Wurzel geben auch hier einen offenkundigen Beweis für die Verschiedenheit des Baues dieser Organe. Epidermis und Rinde des Wurzelträgers stimmen, wie bei anderen Arten, mit den entsprechenden Geweben der Sprosse überein. Die Epidermis ist stark sklerenchymatisch, ebenso die ersten anschließenden Rindenschichten, welche dann nach innen in ein großlumigeres weniger verdicktes Parenchym übergehen. Die der Endodermis angrenzenden Rindenschichten sind wieder kleinlumig und verdickt. Die Endodermis aber und das monarche Leitbündel mit kollateraler Anordnung des Gefäßteiles gleichen den entsprechenden Elementen der Wurzel. . Für diese Endodermis sind Parallelen zu der der Sprosse noch nicht klargelegt, aber die Bündel gestatten Vergleiche mit dem Bündel in den Hypokotylen von Keimpflanzen, aus deren monarchem radiärem Bündel die kollaterale Form leicht abgeleitet werden

1) Mager, Beiträge zur Anatomie der physiologischen Scheiden der Pteridophyten. Marburg 1907 . 
kann. Auch führen ja einige Wurzelträger, z. B. die von der S. Kraussiana und Poulteri, mit den Hypokotylen übereinstimmende Bündel ${ }^{1}$ ). Auch die Wurzel ist, wie bei anderen Arten, namentlich durch ihre beiden äußeren Zellschichten, Epidermis und Hypodermis, von dem Träger abweichend charakterisiert, und diese beiden Schichten knüpfen, an der Basis der Wurzel gleich scharf differenziert, so an die Rinde des Trägers an, daß dessen äußere Schichten frei bleiben und Zeugnis für die endogene Entstehung der Wurzel in ihm ablegen. Die Wände beider peripherischer Zellagen der Wurzel erscheinen, wie auch die der Endodermis, infolge ihrer eigentümlichen Lichtbrechung schwarz. Sie sind, wie Mager durch eingehende Prüfung festgestellt hat, nicht verkorkt, sondern verholzt.

Die Epidermis ist meist kleinzelliger als die Hypodermis und überzieht, wie nur bei dieser Art bekannt, als ein scharf differenziertes Dermatogen die Scheitelkuppe ${ }^{2}$ ).

Auch die Hypodermis tritt als eine gut ausgebildete Sonderschicht auf, welche, im Querschnitt gesehen, einen geschlossenen Zellenkranz von verhältnismäßig großlumigen Zellen darstellt. Eine sekundäre Verdickung, wie an der auch rhizoidlosen Wurzel von S. spinulosa, die ich beschrieben und abgebildet habe, bemerkte ich nicht. Auch diese Schicht wird scheitelwärts frühzeitig differenziert und läßt sich bei dünnen Wurzeln als aus erster perikliner Spaltung der dem Dermatogen angrenzenden Meristemschicht hervorgegangen nachweisen. An die Hypodermis anschließend folgen einige Schichten engerer, mäßig verdickter Rindenzellen, welche in dünnwandige und weitlumige übergehen und dann mit einzelnen verdickten Schichten an die Endodermis angrenzen. Endodermis und Bündelscheide, sowie das Bündel selbst bieten keine Abweichungen dar. Die Endodermis enthält nicht die sekundären Verdickungen, welche ich bei der rhizoidlosen Wurzel von S. spinulosa fand. Mager ${ }^{3}$ ), der diese Wurzel eingehend untersucht hat, erwähnt nicht des endophytischen Pilzes, welcher deutlich in jeder Wurzel zu bemerken ist und wesentliche sekundäre Umgestaltungen in ihr hervorruft. Wenn man vielleicht bei dieser Wurzel zu der Annahme gelangte, daß die Gegenwart des Endophyten das Fehlen der Rhizoide

1) Harvey-Gibson führt auch noch Selagin. delicatissima mit zentroxylischen Bündeln in den Wurzelträgern an, was ich aber nicht bestätigen kann. Genannte Art besitzt in ihren Trägern die gewöhnliche Bündelform.

2) Selag. Preissiana besitzt, wie eine neuere Untersuchung ergab, das gkeiche Wurzelwachstum.

3) Mager, a. a. O., pag. 27. 
veranlasse, so besitzen wir doch an S. Lyallii eine rhizoidlose Wurzel, ohne daß sie zugleich Pilzwurzel ist. Doch will ich noch bemerken, daß ich nur in Gewächshäusern kultivierte Pflanzen untersuchte. Sel. Preissiana, von welcher ich ihrem Standort entstammendes Alkoholmaterial untersuchte, besitzt rhizoidlose Pilzwurzeln mit dem gleichen Scheitelwachstum, wie wir es an den Wurzeln von Sel. Lyallii kennen gelernt haben.

\section{Hauptergebnisse.}

Wie aus der vorstehenden Darstellung folgt, hat die Selaginella Lyallii Spring inmitten der artenreichen Gattung in ihrem abweichenden Bau und in dem Wachstum der vegetativen Organe eine eigenartige Individualität erreicht, welche sich kurz im wesentlichen wie folgt charakterisieren läßt:

Den Vegetationspunkt der Sprosse nehmen Initialen ein, von deren Wachstumstätigkeit alle Gewebe des Scheitels abzuleiten sind. Dieses Scheitelwachstum stimmt mit dem der Sprosse von S. spinulosa und dem von Lycopodium überein.

Die Verzweigungen der Sprosse werden wie dichotomische durch Verbreiterung des Scheitels, Auflösung des Wachstums in der Scheitelmitte und Verlegung desselben auf zwei davon seitliche Stellen eingeleitet. Aber in der Ausführung wird sie durch die frühe Bevorzugung des einen der beiden Auszweigungsäste modifiziert und dadurch nachträglich zu einer monopodialen geprägt. Bei der Verzweigung des Rhizoms erscheint der die Hauptrichtung beibehaltende und das Rhizom fortsetzende Ast als der am wenigsten geförderte, ihn überholt der aufsteigende Wedelast schnell. Bei der Wedelverzweigung dagegen wird der die Hauptrichtung fortsetzende Ast mehr gefördert.

Das Bündel des Rhizoms besteht aus trichterförmig zusanmengeschlossenen und ineinander geschobenen Bandbündelröhren, welche durch Grundgewebe getrennt sind. Bei den seitlichen Abzweigungen des Rhizoms bildet das äußere Bündelrohr Maschen. Querschnitte durch das Rhizom zeigen je nach dessen Stärke ein bis zwei (vielleicht auch mehr) konzentrische, durch Grundgewebe getrennte Bündelringe oder Durchgangsphasen zu solchen. Die Erstlingstracheiden treten an der Peripherie des äußeren Xylemringes auf.

Schwache Rhizome besitzen ein zentrales zylindrisches, noch unfertiges, also ursprüngliches oder zentroxylemisches Bündel. In der einfachsten Form ist ihr Xylem zentral von Phloem und Scheide umgeben. Es erweitert und vervollständigt- sich in dem erstarkenden Rhizom bei stets zentraler Ansetzung der weiteren Bandbündelelemente und darauf 
folgender trichterförmiger Erweiterung zu einem und mehreren konzentrischen Trichterröhrenbündeln.

Die aus den Rhizomen abgezweigten Wedel besitzen in ihrem Stiele eine Anzahl einzelner paralleler Bündel. Die Stiele ganz schwacher Wedel haben nur ein Doppelbündel oder zwei einzelne. Bei Zunahme der Stieldicke nimmt die Zahl der gesonderten, vielfach anastomosierenden Bündel zu und steigt auf 10 und mehr, welche, im Querschnitt gesehen, in drei eine zentrale quadratische Fläche besetzende Reihen angeordnet erscheinen.

Die einfachsten Wedelzweige enden mit einem Doppelbündel oder zwei parallel längs den Blattzeilen verlaufenden Bündeln, welche sich bei einer vegetativen Regeneration zunächst zu einem zentroxylemischen Rhizombündel umbauen.

Epidermis und Rinde haben bei allen Sprossen normalen Bau. Von den oberirdischen Blättern mit bekanntem hochdifferenziertem Bau unterscheiden sich sehr wesentlich die Niederblätter des Rhizoms mit ihrer sehr abweichenden und einfachen Struktur.

Die Wurzelträger werden nur an den Auszweigungen des Rhizoms hervorgebracht und bleiben kurz und einfach. Sie entstehen exogen aus prismatischen Initialen. Ihr kurzes meristematisches Scheitelwachstum wird sehr bald in ein interkalares übergeführt. Während der letzteren Wachstumsweise differenziert sich die Wurzel.

Die Wurzel zeigt in der Scheitelkuppe eine scharfe Gliederung ihres Bildungsgewebes in drei gesonderte Meristeme. Die Wurzelhaube wächst unabhängig vom Wurzelkörper durch ein Kalyptrogen. Den Wurzelkörper selbst überzieht ein scharf differenziertes Dermatogen, wogegen Periblem und Plerom in genetischem Zusammenhange stehen.

Die Verzweigung der Wurzel ist, wie die der Sprosse, modifiziert dichotomisch, wobei in der ungleichen Ausbildung der Wurzelzweige eine scheinbar monopodiale Gestaltung der Verzweigung hervortritt.

Die Wurzeln entspringen nur in den Wurzelträgern, wo sie endogene Entstehung finden. Besonders markiert sich bei ihrer Anlage die Differenzierung des Dermatogens im neuen Wurzelscheitel, für welches größere Zellen des interkalaren Meristems gewonnen und in Form eines Kugelgewölbes angeordnet werden und einheitliche Teilungsfähigkeit erhalten. Mit der Differenzierung des Dermatogens ist auch die der anderen Meristeme und somit die des Wurzelscheitels beendet.

Der Bau der Wurzeln und deren Träger ist normal. Der Hauptunterschied zwischen diesen Organen tritt in dem Bau ihrer Epidermis deutlich hervor. Epidermis und Hypodermis der Wurzel sind gut ausgebildet. Ein Endophyt wurde in der rhizoidlosen Wurzel nicht gefunden. 